\title{
Hip MR Arthrography for Acetabular Labral Tears
}

Tears of the acetabular labrum are an uncommon cause of hip pain. A snapping sound may also accompany labral tears, although this sign is by no means specific for that disorder and may be found with a variety of abnormalities most commonly the snapping of the iliotibial band over the greater trochanter.

\section{IMAGING OF ACETABULAR TEARS}

It is the authors' opinion that the MR assessment of labral tears should be performed as an MR arthrogram, employing an array or surface coil and using higher resolution than that employed for routine MR examinations of the hip MR. The authors also believe that these studies should not be attempted on systems with static field strength $<1$ Tesla, because of the considerable demands placed on these systems to evaluate the labrum, in particular, the need for high resolution and fat saturation. (Not all investigators agree with the necessity of using arthrography, however. At least one investigator maintains that intra-articular contrast is not needed if sufficiently high resolution is employed. A different group of investigators performed MR arthrograms on a group of patients using a 0.5-T system for some patients and a 1.0-T system for others. These investigators did not describe any difference in accuracy between the two systems, although, they did not directly address this issue.) Under fluoroscopic guidance, a small amount of iodinated contrast agent is injected to confirm intra-articular location of the needle. A small amount of dilute Gd-chelate in saline, bupivicaine, and betamethasone is injected into the joint. Bupivicaine, a long-acting anesthetic, is used to confirm the intra-articular origin of the pain, as well as to provide short-term relief of symptoms. Betamethasone, a long-acting steroid, is used to provide somewhat longer-term symptomatic relief. Following the acquisition of a scout sequence in the coronal plane, fat-saturated, $T_{1}$-weighted, fast-spin echo sequences in the transverse, coronal, and sagittal planes are obtained. Fast spin echo (FSE) sequences are used because fat saturation, when used with short $T_{\mathrm{E}}$ FSE sequences, increases the study time much less than when used with short $T_{\mathrm{E}}$ conventional spin echo sequences (CSE). The authors also set the second echo of the echo train in the FSE sequence to two times the minimum $T_{\mathrm{E}}$ as the effective $T_{\mathrm{E}}$ because of reduced blurring compared with use of the first echo. See Kowalchuk et al. (2000) for further discussion of the rationale for using the second echo of a fast-spin echo sequence as well as demonstrating its accuracy for the assessment of knee meniscal tears. To keep this effective $T_{\mathrm{E}}<30 \mathrm{msec}$ while using the second echo of the train requires relatively strong gradients and wide bandwidth. This type of sequence cannot be performed on all systems. In particular, if the gradients are not sufficiently strong, fat-saturated, $T_{1}$-weighted CSE sequences are used and more time is taken to perform the study. Other investigators have advocated the use of 3-D, $T_{1}$-weighted, short $T_{\mathrm{R}}$, gradient sequences. The authors have had no experience with these sequences for this application.

The authors typically use a small FOV (200 to $240 \mathrm{~mm}$ ) and a 256 by 192 acquisition matrix. These are followed by a long $T_{\mathrm{E}}$ FSE sequence with fat saturation, obtained in either the coronal or the transverse plane. This last sequence is primarily used to look for disease in the marrow or surrounding soft tissues. When performed with FSE sequences, this imaging protocol should require $<30 \mathrm{~min}$ from start to finish.

Table A26.2.1 lists the hardware necessary to perform the procedure, along with appropriate parameters. The available gradient strength will depend on the scanner and determines whether to use an FSE sequence or a CSE sequence as described above.

Contributed by J. Bruce Kneeland, Carolyn Kaut Roth, James Garrison, and Anthony Testa

Current Protocols in Magnetic Resonance Imaging (2003) A26.2.1-A26.2.11

Copyright () 2003 by John Wiley \& Sons, Inc.

Hip

A26.2.1

Supplement 8 
Table A26.2.1 Equipment Parameters for MR Arthrography of the Hips for Acetabular Labral Tears

\begin{tabular}{ll}
\hline Coil type & Torso array coil (or similar local coil) \\
Gradient coil strength & $25 \mathrm{mT} / \mathrm{m}$ (or whatever the system permits) \\
Cardiac gating & No \\
Peripheral gating & For safety only \\
Respiratory gating & No \\
Respirator & If required by patient \\
Oxygen & If required by patient \\
Motion cushions & Useful \\
Use of contrast agents & Yes (intra-articular injection performed \\
& with fluoroscopic guidance prior to MR) \\
\hline
\end{tabular}

NOTE: Be sure that technologists and nurses have immediate access to any emergency equipment that may be relevant to a given study, or that may be needed for a particular patient, such as crash carts or oxygen.

\section{Materials}

Betamethasone

Bupivicaine MPF (methyl paraben-free)

Gd-chelate

Iodinated contrast agent

Saline

\section{Set up patient and equipment}

1. Interview (screen) the patient to ensure that he or she has no contraindications such as cardiac pacemakers or other implants containing ferromagnetic materials. Also be sure to find out if the patient has any health conditions that may require the presence of special emergency equipment during the scanning procedure, or necessitate any other precautions. The patient should be questioned as to the location of the pain, in the particular side.

Generally, standard screening forms (APPENDIX 1) are used for all patients scanned in a magnetic resonance system.

The presence of any ferromagnetic metals may be a health hazard to the patient when he or she is inside the magnet, and will also affect the imaging. If in doubt as to the exact composition of the items, it is best to exclude patients with any metal implants; see Shellock (1996) for a discussion of what implants may be safely scanned using magnetic resonance.

Patients may be accompanied into the magnet room by a friend or family member, who can sit in the room during the scan and comfort the patient as needed. This companion must be treated as if he/she was having the MR examination him-or herself to ensure the absence of loose metal objects on the body or clothing. Because of the need for a sterile field, the authors recommend that the companion not be permitted in the fluoroscopic suite. If the companion must be in the fluoroscopic suite to perform the procedure, appropriate radiation protection (i.e., a lead apron) must be worn and the observer is kept at a reasonable distance from the sterile field.

2. If the procedure is a research protocol, have the patient sign any necessary consent forms.

Hip MR Arthrography for Acetabular

Labral Tears

3. Have the patient remove all jewelry and change into a gown to eliminate any metal that might be found in clothing. 
4. Perform a hip arthrogram under fluoroscopic guidance. Inject a small amount of iodinated contrast agent into the hip joint using standard technique to establish the intra-articular location of the needle. Inject into the hip a solution consisting of 0.2 $\mathrm{ml} \mathrm{Gd}$-chelate, $5 \mathrm{ml}$ saline, $1 \mathrm{ml}(6 \mathrm{mg})$ betamethasone, and $5 \mathrm{ml}$ bupivicaine MPF. Pre-mix this solution in a small saline bottle by withdrawing $15 \mathrm{ml}$ from a 20-ml vial and adding the other compounds in order to reduce time in the fluoroscopic suite. Inject the entire $11.2 \mathrm{ml}$ of the solution into the hip followed by an injection of up to $10 \mathrm{ml}$ of saline. This last saline injection may be halted before the full $10 \mathrm{ml}$ is administered if either the patient experiences pain or considerable backpressure is felt.

The patient's assessment of the change in hip pain following the injection of the betamathasone and bupivicaine is used by the referring physician to confirm that intra-articular structures especially the labrum are the cause of pain, as well as to deliver short-term relief from symptoms.

The MPF formulation is used to prevent precipitation with the betamethasone.

5. Take the patient by wheelchair to the MR system.

6. Inform the patient about what will occur during the procedure, what he or she will experience while in the magnet, and how to behave, including the following:

a. If earphones or headphones are used to protect the ears from the loud sounds produced by the gradients, the patient will be asked to wear these, but will be able to communicate with you at any time during the imaging.

b. The patient will be given a safety squeeze-bulb or similar equipment to request assistance at any time (demonstrate how this works).

c. For good results, the patient should not talk, and should avoid or minimize other movement during each scan-i.e., as long as the banging sounds continue. Between scans, talking is allowed in most cases, but should be avoided when comparative positional studies are being performed; the patient will be informed when this is the case.

d. Nevertheless, the patient may call out at any time if he or she feels it necessary.

7. Have the patient lie down on the table with his or her feet toward the machine. Either before or right after the patient lies down in the supine position, set up any triggering devices or other monitoring equipment that is to be used.

8. Center the patient in a torso array coil or other form of dedicated radiofrequency coil at the hip(s) where the key information is desired. Generally, the top of the torso array coil is at the iliac crest and the bottom just below the greater trochanter.

9. Center positioning light at about the midpoint between the anterior superior iliac spine and the symphysis pubis and put him or her into the center of the magnet. This usually falls to the center of the torso array coil.

Once this step has been performed, so long as the patient does not move on the table, the table itself can be moved and then replaced in the same position as before without jeopardizing the positioning of one scan relative to another.

10. Sedation is not needed for routine cases and should be reserved for severe claustrophobics.

\section{Sequence 1: Rapid coronal scout}

11. Run a scout series in the coronal plane using the parameters in Table A26.2.2. (Fig. A26.2.1). 
Table A26.2.2 Coronal Scout

Patient position

Scan type

Imaging plane (orientation)

Central slice or volume center

Echo time $\left(T_{\mathrm{E}}\right)$

Receiver bandwidth (RBW)

Repeat time $\left(T_{\mathrm{R}}\right)$

Flip angle (FA)

Fields of view $\left(\mathrm{FOV}_{\mathrm{x}}, \mathrm{FOV}_{\mathrm{y}}\right)$

Resolution $(\Delta x, \Delta y)$

Number of data points collected $\left(N_{\mathrm{x}}, N_{\mathrm{y}}\right)$

Slice thickness $(\Delta z)$

Number of slices

Slice gap

Number of excitations (NEX)

Number of acquisitions $\left(N_{\text {acq }}\right)$

Swap read and phase encoding

Read direction

Slice locations

Flow compensation

No phase wrap (NPW)

Chemical saturation

Spatial saturation

Scan time
Supine

3-D short $T_{\mathrm{R}}$ gradient echo

Coronal

Halfway between the anterior/superior iliac spine and the symphysis pubis

$1.5 \mathrm{msec}$ (or minimum)

$\pm 32 \mathrm{kHz}$

$6 \mathrm{msec}$ (or minimum)

$30^{\circ}$

$480 \mathrm{~mm}, 480 \mathrm{~mm}$

$1.88 \mathrm{~mm}, 3.75 \mathrm{~mm}$

256, 128

$10 \mathrm{~mm}$

15-20

$0 \mathrm{~mm}$

2

1

No

Right-left

From above iliac crest to below greater trochanter

No

No

No

No

-23-31 sec (depends on size of patient)
Hip MR

Arthrography for Acetabular

Labral Tears

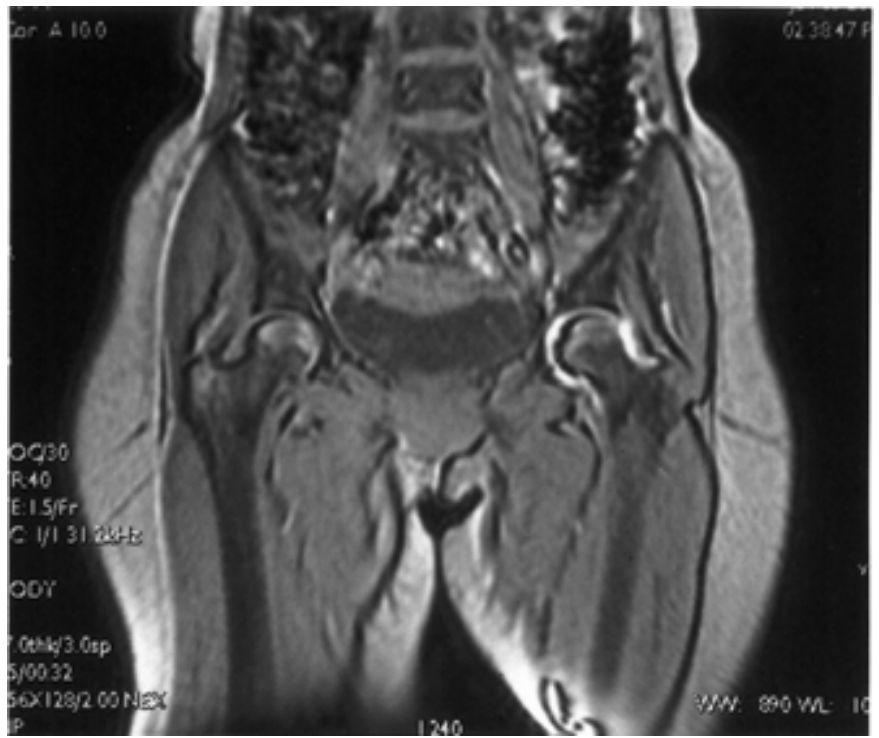

Figure A26.2.1 A representative slice from the coronal series obtained using the parameters listed in Table A26.2.2. 


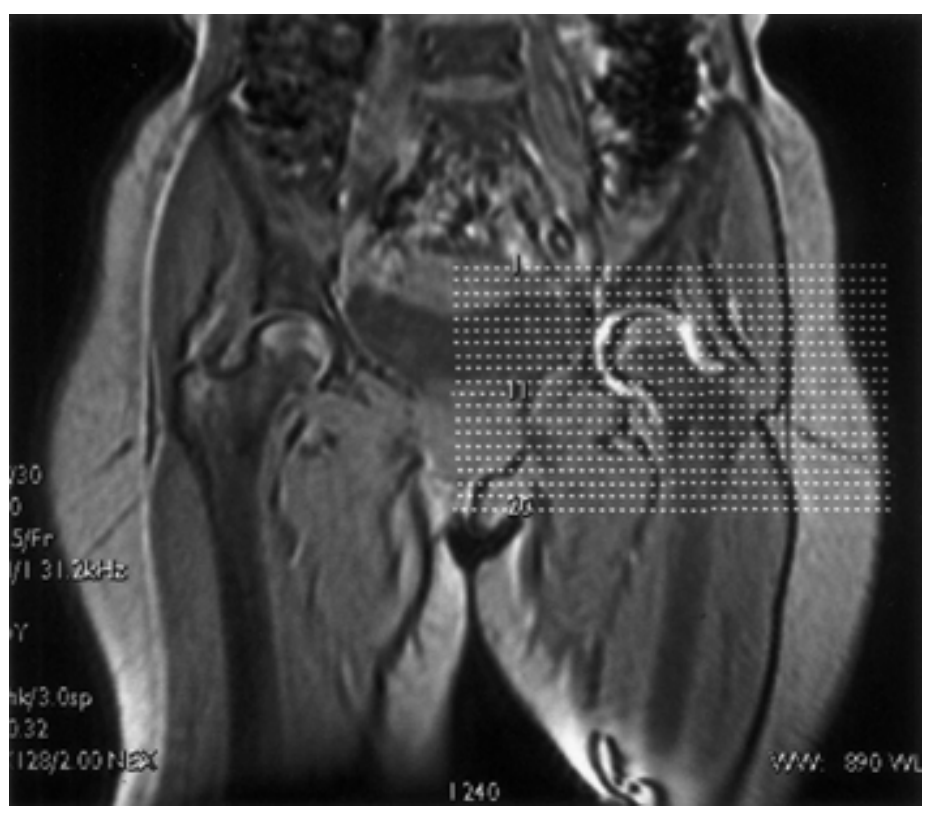

Figure A26.2.2 An image from coronal scout series with locations for the images of both of the following transverse series (sequences 2 and 5 ) indicated.

Table A26.2.3 Transverse Fat-Saturated $T_{1}$-Weighted FSE

\begin{tabular}{ll}
\hline Patient position & Supine \\
Scan type & Fast spin echo \\
Imaging plane (orientation) & Transverse \\
Central slice or volume center & Centered on the hips \\
Echo time $\left(T_{\mathrm{E}}\right)$ & $\sim 20$ msec (second echo of the echo \\
& train set at two times the minimum $T_{\mathrm{E}}$ \\
& as the effective echo time) \\
Receiver bandwidth (RBW) & $\pm 32 \mathrm{kHz}$ \\
Echo train length (ETL) & 4 \\
Repeat time $\left(T_{\mathrm{R}}\right)$ & $600-800$ msec \\
Flip angle (FA) & $90^{\circ}($ or default) \\
Fields of view (FOV, $\left.\mathrm{FOV}_{\mathrm{y}}\right)$ & $200-240 \mathrm{~mm}, 200-240 \mathrm{~mm}$ \\
Resolution $(\Delta x, \Delta y)$ & $0.78-0.94 \mathrm{~mm}, 1.04-1.25 \mathrm{~mm}$ \\
Number of data points collected $\left(N_{\mathrm{x}}, N_{\mathrm{y}}\right)$ & 256,192 \\
Slice thickness $(\Delta z)$ & 4 mm \\
Number of slices & $25-30$ \\
Slice gap & $0.5 \mathrm{~mm}$ \\
Number of excitations $(\mathrm{NEX})$ & 3 \\
Number of acquisitions $\left(N_{\mathrm{acq}}\right)$ & 2 (interleaved series) \\
Read direction & Right-left \\
Slice location & From posterior to the SI (sacroiliac) \\
& joints to anterior to symphysis \\
pubis-include all areas with Gd \\
No phase wrap (NPW) & contrast agent \\
Chemical saturation & Yes \\
Spatial saturation & Yes (fat) \\
Scan time & No \\
\hline
\end{tabular}




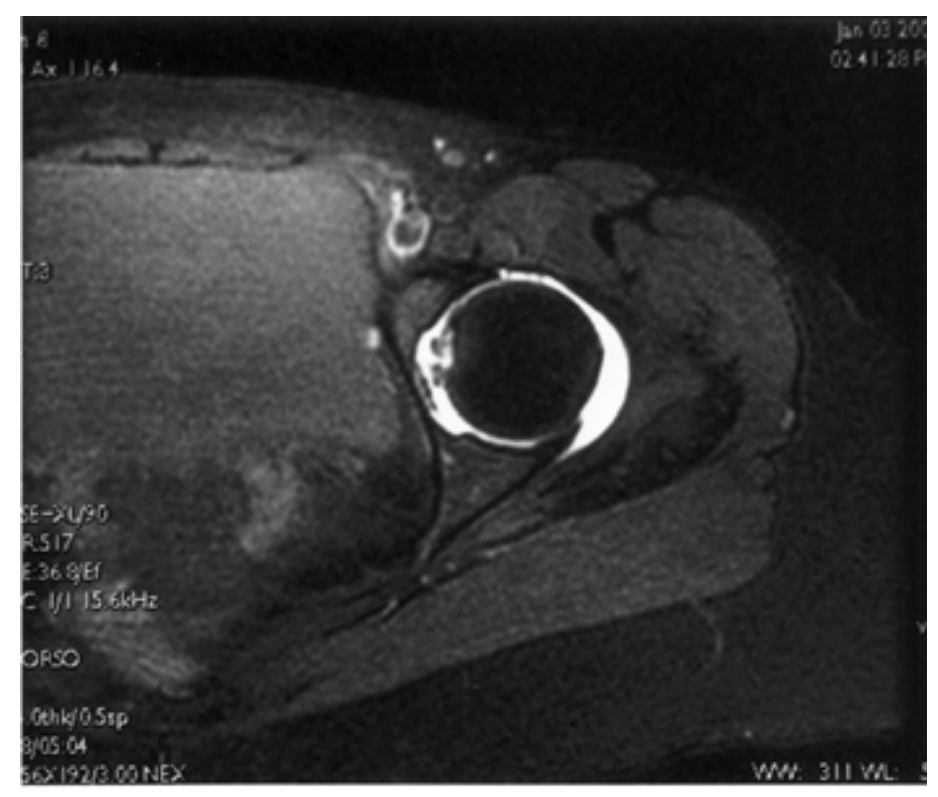

Figure A26.2.3 A representative image from the transverse, fat-saturated, $T_{1}$-weighted FSE sequence obtained with parameters listed in Table A26.2.3.

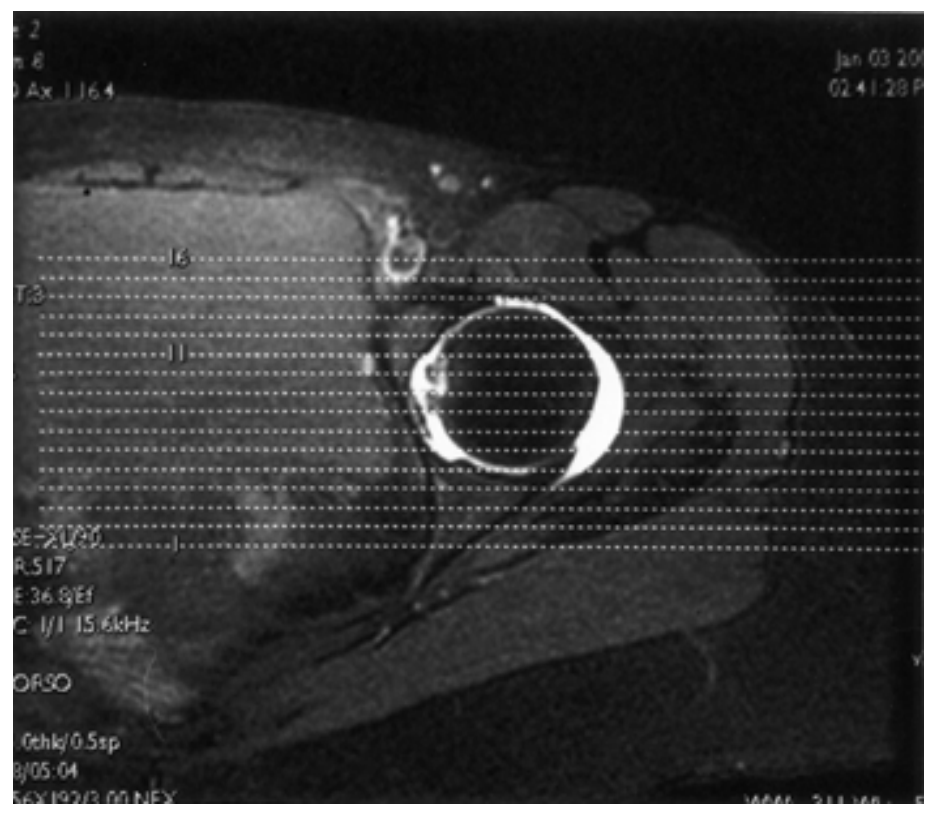

Figure A26.2.4 An image from the transverse, fat-saturated, $T_{1}$-weighted FSE sequence with locations for coronal series (sequence 3 ) indicated.

Hip MR Arthrography for Acetabular

Labral Tears

A26.2.6 
Table A26.2.4 Coronal Fat-Saturated $T_{1}$-Weighted FSE

Patient position

Scan type

Imaging plane (orientation)

Central slice or volume center

Echo time $\left(T_{\mathrm{E}}\right)$

Receiver bandwidth (RBW)

Echo train length (ETL)

Repeat time $\left(T_{\mathrm{R}}\right)$

Flip angle (FA)

Fields of view $\left(\mathrm{FOV}_{x}, \mathrm{FOV}_{y}\right)$

Resolution $(\Delta x, \Delta y)$

Number of data points collected $\left(N_{\mathrm{x}}, N_{\mathrm{y}}\right)$

Slice thickness $(\Delta z)$

Number of slices

Slice gap

Number of excitations (NEX)

Number of acquisitions $\left(N_{\text {acq }}\right)$

Read direction

Slice location

No phase wrap (NPW)

Chemical saturation

Scan time

\section{Supine}

Fast spin echo

Coronal

Centered on the hip

$\sim 20 \mathrm{msec}$ (second echo of the echo train set at two times the minimum $T_{\mathrm{E}}$ as the effective echo time)

$\pm 32 \mathrm{kHz}$

4

600-800 msec

$90^{\circ}$ (or default)

200-240 mm, 200-240 mm

0.78-0.94 mm, $1.04-1.25 \mathrm{~mm}$

256, 192

$4 \mathrm{~mm}$

25-30

$0.5 \mathrm{~mm}$

3

2 (interleaved series)

Superior-inferior

From just anterior to the hip to just posterior

Yes

Yes (fat)

$\sim 3$ min (depends on $T_{\mathrm{R}}$ )

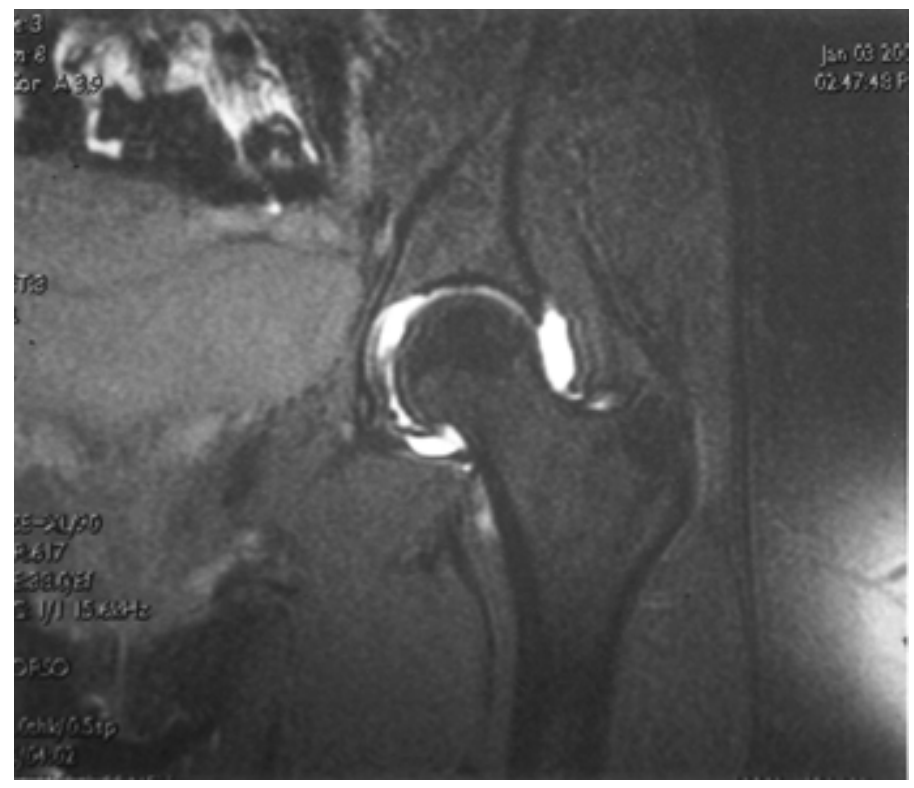

Figure A26.2.5 A representative image from the coronal, fat-saturated, $T_{1}$-weighted FSE sequence obtained with parameters listed in Table A26.2.4. 


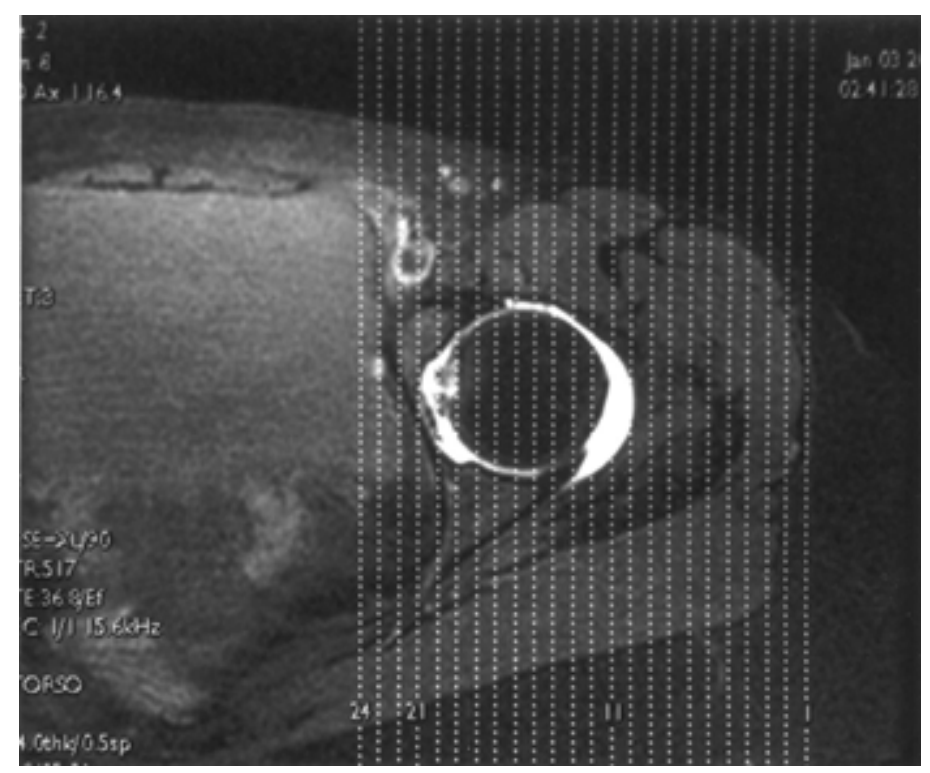

Figure A26.2.6 An image from the transverse, fat-saturated, $T_{1}$-weighted FSE sequence with locations for sagittal series (sequence 4 ) indicated.

Table A26.2.5 Sagittal Fat-Saturated $T_{1}$-Weighted FSE

\begin{tabular}{|c|c|}
\hline Patient position & Supine \\
\hline Scan type & Fast spin echo \\
\hline Imaging plane (orientation) & Sagittal \\
\hline Central slice or volume center & Centered on the hip \\
\hline Echo time $\left(T_{\mathrm{E}}\right)$ & $\begin{array}{l}\sim 20 \text { msec (second echo of the echo } \\
\text { train set at two times the minimum } T_{\mathrm{E}} \\
\text { as the effective echo time) }\end{array}$ \\
\hline Receiver bandwidth (RBW) & $\pm 32 \mathrm{kHz}$ \\
\hline Echo train length (ETL) & 4 \\
\hline Repeat time $\left(T_{\mathrm{R}}\right)$ & $600-800 \mathrm{msec}$ \\
\hline Flip angle (FA) & $90^{\circ}$ (or default) \\
\hline Fields of view $\left(\mathrm{FOV}_{\mathrm{x}}, \mathrm{FOV}_{\mathrm{y}}\right)$ & $200-240 \mathrm{~mm}, 200-240 \mathrm{~mm}$ \\
\hline Resolution $(\Delta x, \Delta y)$ & $0.78-0.94 \mathrm{~mm}, 1.04-1.25 \mathrm{~mm}$ \\
\hline Number of data points collected $\left(N_{\mathrm{x}}, N_{\mathrm{y}}\right)$ & 256,192 \\
\hline Slice thickness $(\Delta z)$ & $4 \mathrm{~mm}$ \\
\hline Number of slices & $25-30$ \\
\hline Slice gap & $0.5 \mathrm{~mm}$ \\
\hline Number of excitations (NEX) & 3 \\
\hline Number of acquisitions $\left(N_{\text {acq }}\right)$ & 2 (interleaved series) \\
\hline Read direction & Superior-inferior \\
\hline Slice location & $\begin{array}{l}\text { From just medial to the acetabulum to } \\
\text { the lateral margin of the surrounding } \\
\text { muscles }\end{array}$ \\
\hline No phase wrap (NPW) & Yes \\
\hline Chemical saturation & Yes (fat) \\
\hline Scan time & $\sim 3$ min (depends on $T_{\mathrm{R}}$ ) \\
\hline
\end{tabular}




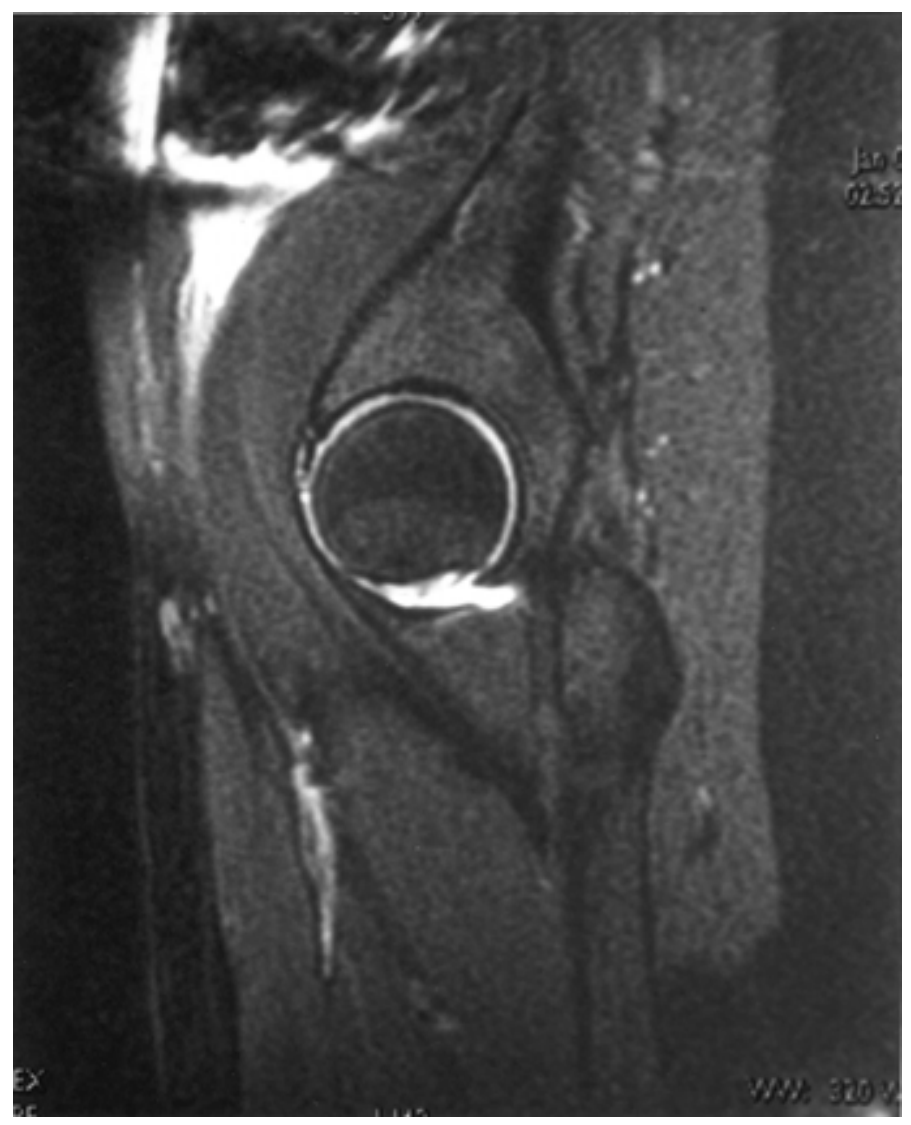

Figure A26.2.7 A representative image from the sagittal, fat-saturated, $T_{1}$-weighted FSE sequence obtained with parameters listed in Table A26.2.5.

Sequence 2: Transverse $T_{1}$-weighted, fat saturated, FSE

12. Use scout (coronal) images to prescribe transverse images (Fig. A26.2.2). Run a transverse, fat-saturated, $T_{1}$-weighted FSE sequence using the parameters given in Table A26.2.3 (Fig. A26.2.3).

The rationale for using FSE sequences with the second echo of the echo train set at two times the minimum $T_{E}$ as the effective $T_{E}$ was explained above. If the gradient strength is not sufficient to permit a $T_{E}$ of $\leq 30 \mathrm{msec}$, a conventional spin echo sequence with the minimal $T_{E}$ achievable on the system should be employed. $T_{R}$ must be sufficiently short to ensure $T_{1}$-weighting.

\section{Sequence 3: Coronal $T_{1}$-weighted, fat-saturated, FSE}

13. Use previously obtained transverse images to prescribe coronal images (Fig. A26.2.4). Run a coronal, fat-saturated, $T_{1}$-weighted FSE sequence using the parameters given in Table A26.2.4 (Fig. A26.2.5).

This sequence is identical to sequence 2 except for the orientation.

\section{Sequence 4: Sagittal $T_{1}$-weighted, fat-saturated, FSE}

14. Use previously obtained transverse images to prescribed sagittal images (Fig. A26.2.6). Run a sagittal, fat-saturated, $T_{1}$-weighted FSE sequence using the parameters given in Table A26.2.5 (Fig. A26.2.7). 
Table A26.2.6 Transverse $T_{2}$-Weighted Fat-Saturated Fast-Spin Echo

\begin{tabular}{ll}
\hline Patient position & Supine \\
Scan type & 2-D fast-spin echo \\
Imaging plane (orientation) & Transverse \\
Central slice or volume center & Centered on the hip \\
Echo time $\left(T_{\mathrm{E}}\right)$ & $90 \mathrm{msec}$ \\
Receiver bandwidth (RBW) & $\pm 16 \mathrm{kHz}$ \\
Echo train length (ETL) & 8 \\
Repeat time $\left(T_{\mathrm{R}}\right)$ & $6000 \mathrm{msec}$ \\
Flip angle (FA) & $90^{\circ}$ (or default) \\
Fields of view (FOV, $\left.\mathrm{FOV}_{\mathrm{y}}\right)$ & $380 \mathrm{~mm}, 380 \mathrm{~mm}$ \\
Resolution $(\Delta x, \Delta y)$ & $1.48 \mathrm{~mm}, 1.98 \mathrm{~mm}$ \\
Number of data points collected $\left(N_{\mathrm{x}}, N_{\mathrm{y}}\right)$ & 256,192 \\
Slice thickness $(\Delta z)$ & $5 \mathrm{~mm}$ \\
Number of slices & $30-40$ (depends on $\left.T_{\mathrm{R}}\right)$ \\
Slice gap & $1 \mathrm{~mm}$ \\
Number of excitations $(\mathrm{NEX)}$ & 1 \\
Number of acquisitions $\left(N_{\mathrm{acq}}\right)$ & 2 \\
Swap read and phase encoding & Yes \\
Read direction & Anterior-posterior \\
Slice location & From above iliac crests to below lesser \\
& trochanters \\
No phase wrap (NPW) & Yes \\
Chemical saturation & Yes (fat) \\
Spatial saturation & Yes (superior and inferior) \\
Scan time & $\sim 5$ min (depends on $T_{\mathrm{R}}$ ) \\
\hline
\end{tabular}

\section{Sequence 5: Transverse, fat-saturated, $T_{2}$-weighted FSE}

15. Use scout (coronal) images to prescribe transverse images (Fig. A26.2.2). Run a transverse, fat-saturated, $T_{2}$-weighted FSE sequence using the parameters given in Table A26.2.6. (A representative image can be seen as Fig. A26.1.7.)

\section{COMMENTARY}

\section{Background Information}

Acetabular labral tears are an uncommon but significant cause of hip pain that is amenable to surgical treatment. They are often accompanied by a snapping sound but this is also seen with other disorders of the hip.

\section{Critical Parameters and Troubleshooting}

The authors believe that the use of a highfield (i.e., $\geq 1 \mathrm{~T}$ ) system and MR arthrography in conjunction with surface or array coils to obtain the highest possible resolution are important factors to demonstrate labral abnormalities. However, there have been no studies to prove that any of these factors are critical to obtain accurate diagnoses.
The rationale for the use of the second echo of the echo train for the short- $T_{\mathrm{E}}$, fat-saturated, FSE sequence with this second echo set to the smallest value possible (i.e., two times the minimum echo) is discussed above. Note that the authors use a 32-kHz bandwidth to help achieve this minimum effective $T_{\mathrm{E}}$. If the $T_{\mathrm{E}}$ cannot be kept at $\leq 30 \mathrm{msec}$, it is probably better to used a conventional spin echo sequence.

The number of excitations will depend on the particular coil used, the FOV, the bandwidth, and the size of the patient. For heavy-set patients, choose the largest FOV in the given range $(240 \mathrm{~mm})$ or even increase it up to 280 $\mathrm{mm}$. An increased number of excitations (i.e., more than the NEX $=3$ given above) may be used in conjunction with, or in place of, the 


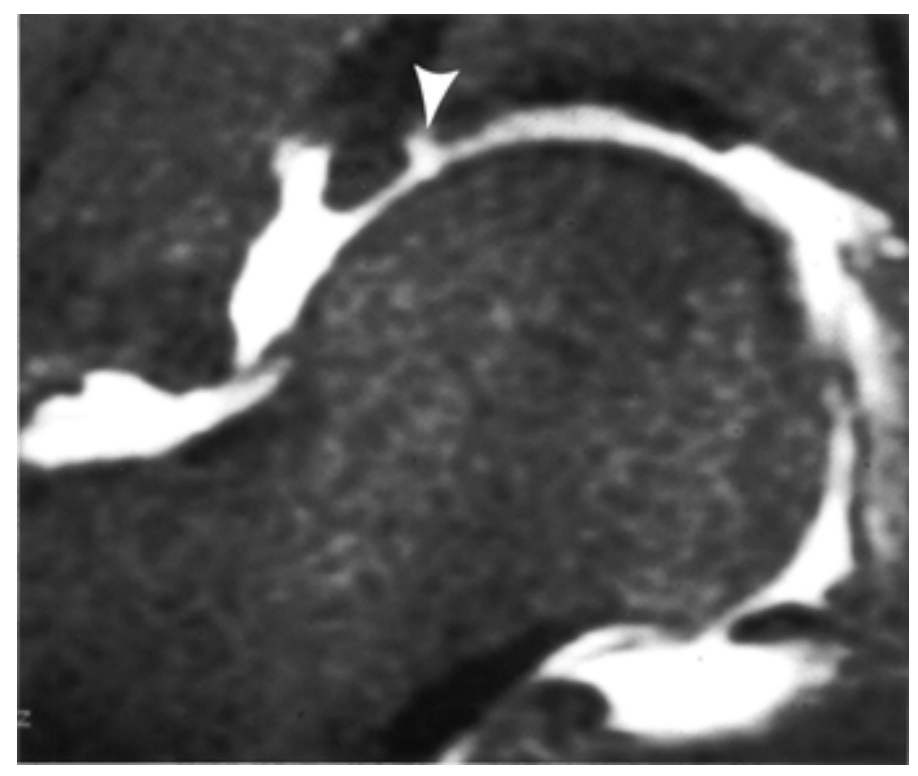

Figure A26.2.8 A coned down, coronal fat-saturated, $T_{1}$-weighted FSE image of the right hip demonstrates the presence of a tear of the superior acetabular labrum (arrowhead).

increased FOV. The technical factors given above work well on the system described in this unit but may have to be altered for different systems.

\section{Anticipated Results}

A small number of cases of surgically confirmed acetabular labral tears have been reported in the radiology literature (Czerny et al., 1999). The anterosuperior segment of the labrum is the region most commonly involved by a tear. Multiple different appearances of tears diagnosed with MR arthrography have been described and include absence, fragmentation, linear defects, clefts, and intrasubstance high signal (Fig. A26.2.8). It is of interest that similar appearing morphologic abnormalities have been described in the labrum on nonarthrographic studies in asymptomatic subjects. They are noted to occur with increased frequency in older subjects and most commonly in the anterosuperior segment. Because these subjects were not studied with arthrography, the relationship between these findings and those seen at arthrography is unclear. Nev- ertheless, it does cast some doubt on the clinical significance of what have been considered to represent labral tears.

\section{Literature Cited}

Czerny, C., Hofmann, S., Urban, M., Tschauner, C., Neuhold, A., Pretterklieber, M., Recht, M.P., and Kramer, J. 1999. MR arthrography of the adult acetabular capsular-labral complex: Correlation with surgery and anatomy. A.J.R. 173:345-349.

Kowalchuk, R., Kneeland, J.B., Dalinka, M.R., Siegelman, E.S., and Dockery, W.D. 2000. MRI of the knee: Valve of short echo time fast spinecho using high performance gradients versus conventional spin-echo for the detection of meniscal tears. Skeletal Radiol. 29:520-524.

Shellock, F.G. 1996. Pocket Guide to MR Procedures and Metallic Objects. Lippincott-Raven, Philadelphia.

Contributed by J. Bruce Kneeland, Carolyn Kaut Roth, James Garrison, and Anthony Testa

University of Pennsylvania

Philadelphia, Pennsylvania 
